\title{
Über Oxydation durch elektrolytisch abgeschiedenes Fluor.
}

\author{
Von
}

F. W. SkirRow.

Wenn man die Reihe der Entladungsspannungen betrachtet,

$$
\mathrm{O}^{\prime \prime}=1.08 ; \mathrm{Cl}^{\prime}=1.4 ; \mathrm{OH}^{\prime}=1.67,
$$

so ist ersichtlich, dafs bei Einleiten von Chlor in Wasser Sauerstoff sich entwickeln mufs. Diese Entwickelung wird jedoch, entsprechend der geringen Konzentration und der kleinen Bildungsgeschwindigkeit der Sauerstoffionen, nur sehr gering sein können.

Würde man dagegen einen Stoff hinzufügen, dessen Lösungstension noch über dem Entladungspotential der $O \mathrm{H}^{\prime}$-Ionen liegt, so würde die Sauerstoffentwickelung bedeutend vermehrt erscheinen. Dies ist nach den Versuchen von Morssan der Fall bei der Einwirkung von Fluor. Es entwickelt sich dabei Sauerstoff und zwar in der dem hohen Bildungspotential entsprechenden aktiven Form als Ozon.

Sind nun in einer Lösung, in welcher Sauerstoff anodisch entwickelt wird, oxydierbare Substanzen zugegen, so hängt die oxydierende Wirkung des Sanerstoffs ab von dem Potential, bei welchem der Sauerstoff entsteht. ${ }^{1}$

Daraus ist zu schliefsen, dafs die weitgehendste Einwirkung auf oxydable Substanzen bei der Elektrolyse durch sekundäre Einwirkung entladener Fluorionen erzeugt wird.

Dieser Schlufs sollte im folgenden zunächst an einigen anorganischen Beispielen geprüft werden.

1 Nernst, Ber. deutsch. chem. Ges. 30, 1547. 


\section{Versuchsanordnung.}

Die Versuche wurden in folgender Weise ausgeführt. In zwei gleich grolsen Platinschalen wurde ein bestimmtes Volum der zu untersuchenden Lösung hineingebracht und mit Flufssäure bezw. Schwefelsäure zum gleichen Säuregehalt angesäuert.

Diese und ein Präzisions-Ampèremeter wurden hinter einander in einen Stromkreis geschaltet. Die Schalen wurden als Anode benutzt, während als Kathode eine Spule von Platindraht diente.

Nachdem eine bestimmte Elektrizitätsmenge hindurch gegangen war, wurde die oxydierte Menge bestimmt und die prozentische Ausbeute (d. h. Prozent-Sauerstoff benutzt zur Oxydation zu Gesamt-Sauerstoff nach dem FARADAY'schen Gesetz) berechnet.

Es wurde noch die Möglichkeit geprüft, ob vielleicht unter diesen Bedingungen eine Bildung von Hydroperoxyd oder Überschwefelsäure stattfindet. Ein Versuch wurde dazu in der Weise gemacht, dals verdüunte Schwefelsäure mit Zusatz von Flufssäure bezw. Schwefelsäure elektrolysiert wurde. In keinem Falle aber konnte eine solche Bildung nachgewiesen werden.

In einigen Fällen wurden Versuche gemacht, um die Anodenspannung mittels konstanter Cadmiumelektrode unmittelbar zu bestimmen. Es ist mir aber nicht gelungen, konstante Werte zu erzielen.

\section{0xydation von Chromsalzen.}

Hier wurde eine Lösung von Chromsulfat genommen und mit Flufssäure bezw. Schwefelsäure angesäuert. Durch Elektrolyse entsteht an der Anode Chromsäure, welche mittels Jodkalium und Thiosulfat besirimt wurde. Die Versuchsbedingungen wurden möglichst variiert und die Resultate sind in der Tabelle 1 mitgeteilt.

Tabelle 1.

\begin{tabular}{|c|c|c|c|c|c|c|}
\hline $\begin{array}{c}\text { Konz. } \\
\mathrm{Cr}_{2}\left(\mathrm{SO}_{4}\right)_{3} \\
\text { norm. }\end{array}$ & $\begin{array}{c}\text { Konzentr. } \\
\text { Säure } \\
\text { norm. }\end{array}$ & $\begin{array}{c}\text { Zeit } \\
\text { in } \\
\text { Minuten }\end{array}$ & $\begin{array}{l}\text { Strom } \\
\text { in } \\
\text { Amp. }\end{array}$ & $\begin{array}{c}\text { Elektr. } \\
\text { Menge } \\
\text { (Coulomb) }\end{array}$ & $\begin{array}{l}\% \text { A } \\
\text { Fluls- } \\
\text { säure }\end{array}$ & $\begin{array}{l}\text { sbeute } \\
\begin{array}{c}\text { Schwefel- } \\
\text { säure }\end{array}\end{array}$ \\
\hline 50 & 2.54 & 40 & 0.08 & 192 & 22.34 & 8.8 \\
\hline 50 & 2.54 & 40 & 0.2 & 480 & 15.8 & 9.5 \\
\hline 50 & 2.54 & 18 & 0.2 & 216 & 19.9 & 10.8 \\
\hline 50 & 2.54 & 25 & 0.08 & 120 & 36.7 & 19.0 \\
\hline 50 & 2.54 & 25 & 0.08 & 120 & 37.6 & 19.0 \\
\hline 50 & 2.54 & 10 & 0.08 & 48 & 49.8 & 26.4 \\
\hline 50 & 1.27 & 10 & 0.08 & 48 & 32.6 & 14.1 \\
\hline 10 & 2.56 & 10 & 0.08 & 48 & 70.0 & 28 \\
\hline
\end{tabular}


Man sieht, obschon die Ausbeute stark von der Versuchsdauer, der Stromdichte und der Konzentration abhängt, ist doch immer die Ausbente in der Flufssäurelösung sehr viel grölser als in der Schwefelsäurelösung.

\section{Oxydation von Manganosalzen.}

Für diese Versuche wurde eine Lösung von Mangansulfat ungefähr zehntelnormal angewandt. Es stellte sich heraus, dafs in der Schwefelsäurelösung Braunstein ausgeschieden wird, während in der Flufssäurelösung eine braune Lösung sich bildete. Diese braune Lösung wurde durch Kochen gefällt; mit $\mathrm{NH}_{4} \mathrm{~F}$ gab sie einen rosenroten Niederschlag. Man kann das Produkt also mit Wahrscheinlichkeit als $\mathrm{MnF}_{4}$ betrachten.

Zuerst ist der Versuch, Permanganat daraus zu bekommen, ohne Erfolg geblieben. Die Ursache davon, wie es sich später herausstellte, war die, dafs kein Permanganat dauernd bestehen konnte, bis alles Manganosalz beseitigt war. Dann begann sich plötzlich Permanganat zu bilden und in einigen Minuten war Permanganat reichlich vorhanden. Dafs es wirklich Permanganat war, wurde mit Hilfe des Absorptionsspektrums immer nachgewiesen. Der Vorgang mit HF-Lösung verlief also in zwei Stufen. 1. Bildung von $\mathrm{MnF}_{4}$. 2. Oxydation von $\mathrm{MnF}_{4}$ zu Permanganat. Bei diesem Versuche wurde es, um die Reduktion an der Kathode zu verhindern, vorteilhaft gefunden, nur eine Spitze von Platin als Kathode anzuwenden.

Zunächst seien einige Resultate mitgeteilt, die innder ersteri Stufe erhalten wurden.

Tabelle 2.

$n / 10 \mathrm{MnSO}_{4}$

\begin{tabular}{c|c|c|c|c|c}
\hline $\begin{array}{l}\text { Konz. } \\
\text { Säure } \\
\text { norm. }\end{array}$ & $\begin{array}{c}\text { Zeit } \\
\text { in } \\
\text { Minuten }\end{array}$ & $\begin{array}{c}\text { Stromstärke } \\
\text { in } \\
\text { Amp. }\end{array}$ & $\begin{array}{c}\text { Elekr. } \\
\text { Menge } \\
\text { in Coulomb }\end{array}$ & HF & $\mathrm{H}_{2} \mathrm{SO}_{4}$ \\
\hline 0.4 & 74 & 0.1 & 444 & 54.8 & 36.1 \\
1.2 & 50 & 0.2 & 600 & 37.9 & 16.3 \\
1.2 & 20 & 0.9 & 1080 & 30.87 & 8.73 \\
1.2 & 10 & 0.9 & 540 & 42.9 & 15.2
\end{tabular}

Aus dieser Tabelle geht deutlich hervor, dafs unter den verschiedensten Bedingungen die Ausbeute in der Flufssäurelösung ganz erheblich grölser ist als in der Schwefelsäurelösung. 
Ferner wurde die Elektrolyse so weit geführt, dafs Permanganat in der Flufssäurelösung reichlich vorhanden war, dann war in der Schwefelsäurelösung keine Spur von Permanganat nachzuweisen, d. h. in der Schwefelsäurelösung war noch nicht alles Manganosalz in Braunstein übergeführt. Dals diese Auffassung richtig ist, wird auch durch folgenden Versuch weiter bestätigt. - In verdünnter Schwefelsäure und in verdünnter Flulssäure wurde reiner Braunstein suspendiert und dann werden die beiden hinter einander elektrolysiert. Nach einigen Minuten wurde in beiden Fällen Permanganat gebildet, doch war sofort zu bemerken, dals in der Flufssäure erheblich mehr Permanganat gebildet wurde, wie in der Schwefelsäurelösung. ${ }^{1}$

Es wurden aufserdem Versuche gemacht, um die stufenweise Oxydation ron Mangan in Flufssäurelösung näher zu studieren.

Aus Tabellen 1 und 2 sieht man gleich, dafs bei gleicher Stromdichte die Ausbeute mit zunehmender Zeit immer abnimmt. Es lag der Gedanke nahe, dals, wenn eine neue Reaktion plötzlich eintrat, eine erhöhte Ausbeute stattfinden würde. Es wurden also eine Reihe von Elektrolysen bei gleicher Konzentration und Stromdichte ausgeführt, um die Beziehung zwischen der Zeit und der Ausbeute zu ermitteln. Es wurde in folgender Weise verfahren. Zwei Schalen mit genau derselben Lösung wurden hinter einander elektrolysiert. Nach bestimmter Zeit wurde eine Schale ausgeschaltet und der Inhalt titriert. Die andere wurde dann zehn Minuten länger noch elektrolysiert und dann titriert. In dieser Weise konnte man die Ausbeute in einem bestimmten Zeitintervall berechnen.

Die Ergebnisse sind in Tabelle 3 mitgeteilt.

Tabelle 3.

$\begin{array}{cr}\text { Zeitintervall (10 Minuten) } & \text { Ausbeute in \% } \\ 1 & 42.9 \\ 2 & 18.9 \\ & \text { Permanganat eben gebildet } \\ 3 & 34.3 \\ 4 & 12.1 \\ \text { Permanganat reichlich vorhanden. }\end{array}$

${ }^{1}$ Im Laufe dieses Versuches sind die Beobachtungen von Morse und von Reckunghausen und anderen, Ber. deutsch. chem. Ges. 30, 48, dals neutrales Kaliumpermanganat durch Braunstein reduziert wird bestätigt worden. Die Lösung bleibt dabei neutral und daf's Gesamtkalium wird in Niederschlag erhalten. 
Wie man sieht, fällt die Ausbeute mit der Zeit rasch ab. Sobald aber Permanganat gebildet wird, steigt die Ausbeute plötzlich, um wieder rasch zu sinken.

\section{Oxydation von Kobaltsalzen.}

Vor kurzem hat Herr Dr. CoEHs ${ }^{1}$ gezeigt, dafs, wenn man eine neutrale Lösung von einem Kobaltsalz elektrolysiert, man an der Anode einen schwarzen Überzug von $\mathrm{CO}_{2} \mathrm{O}_{3}$ erhält. Diese Reaktion wird aber durch Säurezusatz vollkommen gehemmt.

Es fragt sich nun, ob die oxydierende Kraft in einer Flufssäurelösung grofs genug wird, um diese hemmende Wirkung zu besiegen; und in der That hat der Versuch gezeigt, dafs, wenn man eine Flufssäurelösung von Kobalt elektrolysiert, ein schwarzer Überzug an der Anode sich ausscheidet, welcher durch Analyse als $\mathrm{Co}_{2} \mathrm{O}_{3}$ sich erwies. In einer gleich sauren Schwefelsäurelösung wurde keine Spur von $\mathrm{Co}_{2} \mathrm{O}_{3}$ gebildet.

Sobald man den Strom unterbricht, geht dieser Überzug schnell in Lösung und zwar wieder als Oxydulsalz.

\section{Über die Oxydation von Kohlensäure.}

Es wurde auch untersucht, ob man bei dieser Methode die Überkohlensäure von CoNSTAM und von $\mathrm{H}_{\mathrm{ANSEN}}{ }^{2}$ bekommen konnte. Es wurde somit ein Strom von Koblensäure in eine Flufssäurelösung, welche in einer Kältemischung sich befand, eingeleitet und gleichzeitig elektrolysiert.

Est stellt sich heraus, dafs auf diese Weise keine Spur von Überkohlensäure gebildet wird.

Dieses Resultat spricht sehr für die Auffassung von Constam und von HaNSEN über die Bildungsweise von Überkohlensäure, dås zwei ihrer Elektrizität beraubte $\mathrm{HCo}_{3}$-Ionen zu elektrisch neutralem $\mathrm{H}_{2} \mathrm{C}_{2} \mathrm{O}_{6}$ zusammentreten, ganz ähnlich wie z. B. zwei Chlorionen zu einem neutralen Chlormoleküle zusammentreten.

Auch konnte, wie früher erwähnt, wenn man ein Gemisch von Schwefelsäure und Flufssäure elektrolysiert, keine Überschwefelsäure nachgewiesen werden.

1 Z. anorg. Chem. 33 (1902), 9.

- Zeitschr. f. Elektrochem. 3 (1896), 137. 


\section{Versuche mit organischen Substanzen.}

Qualitative Versuche zeigten, dals Benzol und Naphthalin in Flufssäurelösung merklich oxydiert werden und zwar schneller, wie in Schwefelsäurelösung. Was aber dabei entsteht, habe ich nicht Gelegenheit gehabt, zu untersuchen, doch scheint es jedenfalls nicht Phenol zu sein.

Auf organischem Gebiete scheint die Anwendung der mitgeteilten Oxydationsmittel besonders aussichtsvoll zu sein. Wegen Abreise aber mulste diese Untersuchung schon jetzt abgeschlossen werden.

Die bisherigen Versuche haben den unzweideutigen Beweis dafür erbracht, dals die sekundäre Einwirkung entladener Fluorionen auf oxydable Substanzen eine energischere Oxydationswirkung herbeiführt, als sich in Lösungen erreichen lälst, die keine Fluorionen enthalten.

Es sei mir an dieser Stelle gestattet, Herm Prof. Dr. Nernst und Herrn Privatdozent Dr. Coens für die Anregung zu dieser Arbeit und ihre mir stets freundlich erteilten Ratschläge meinen verbindlichsten Dank auszusprechen.

Göttingen, Institut für plyysikalische Chemie, August 1902.

Bei der Redaktion eingegangen am 10. August 1902. 